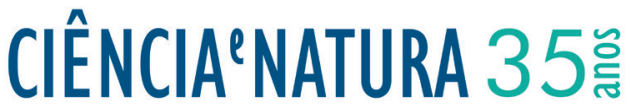

\section{Análise interdecadal e sazonal de ciclones extratropicais sobre a costa sul brasileira de 1957 a 2010}

\author{
Interdecadal and seasonal analysis of extratropical cyclones over southern Brazilian \\ coast from 1957-2010
}

\author{
Maurício Granzotto Mello¹, Michel Nobre Muza² e Mário Francisco Leal Quadro² \\ ${ }^{1}$ Centro de Previsão do Tempo e Estudos Climáticos - Instituto Nacional de Pesquisas Espaciais, CPTEC/INPE; \\ Cachoeira Paulista, SP \\ ${ }^{2}$ Curso Técnico de Meteorologia, Departamento Acadêmico de Saúde e Serviços, DASS/IFSC; Florianópolis, SC
}

\begin{abstract}
Resumo
Este trabalho apresenta a ocorrência e o tempo de vida dos ciclones extratropicais na costa do Brasil, apresentando uma análise sazonal e interdecadal. Os dados dos eventos de ciclones extratropicais foram obtidos através de um esquema de deteç̧ão de sua trajetória a partir de dados da reanálise 1 do NCEP. No presente trabalho, foi elaborado uma seleção da ocorrência de ciclones com persistência igual ou superior a 24 horas, para o período de 1957 a 2010, em uma área correspondente a latitudes de $15^{\circ} \mathrm{S}$ a $45^{\circ} \mathrm{S}$ e longitudes de $20^{\circ} \mathrm{W}$ a $60^{\circ} \mathrm{W}$. O comparativo interdecadal e sazonal proposto está baseado em pesquisas recentes, que sugeriram modulação da variabilidade interdecadal nas séries temporais de dados meteorológicos. Durante este período de 54 anos, a estação com maior ocorrência de ciclones foi o inverno, com 822 eventos. Porém, analisando-se a série temporal em perídos distintos é possível identificar a variabilidade interdecadal e sazonal.
\end{abstract}

Palavras-chave: ciclone extratropical, tempo de vida, análise, sazonal, interdecadal, Brasil.

\begin{abstract}
This work presents the occurrence and the lifetime of extratropical cyclones over the coast of Brazil, presenting a seasonal and interdecadal analysis. The data of extratropical cyclones events were obtained through a detection scheme for trajectory data from NCEP reanalysis-1. In the current work, was made a selection of cyclones with persistence equal or major than 24 hours, for the period 1957-2010, in an area correspondent to $15^{\circ} \mathrm{S}$ to $45^{\circ} \mathrm{S}$ latitude and $20^{\circ} \mathrm{W}$ to $60^{\circ} \mathrm{W}$ of longitude. The interdecadal and seasonal proposed comparative is based in recent research of interdecadal variability. During this 54 years period, winter was the season with most occurrence of cyclones, there was 822 events. However, analyzing the time series in distinct periods is possible identify the interdecadal and seasonal variability.
\end{abstract}

Keywords: extratropical cyclone, lifetime, analysis, sazonal, interdecadal, Brazil. 


\section{Introdução}

A influência dos Andes no deslocamento de anticiclones e na formação de ciclones extratropicais no leste da América do Sul foi estudada por Gan e Rao (1994). Os mecanismos dinâmicos que favorecem a formação dos ciclones extratropicais são um cavado de níveis médios e a disponibilidade de umidade na costa sul do Brasil (Seluchi, 1995). Reboita et al. (2012) mostraram duas regiões favoráveis a formação de ciclones sendo uma na costa sul e outra na região sudeste.

Murray e Simmonds (1991) desenvolveram um esquema numérico para detectar a trajetória de ciclones extratropicais para o Hemisfério Sul a partir de dados de reanálise. O esquema numérico foi testado com dados observados em distribuição irregular sobre a Terra e com dados observados dispostos em pontos de grade. Bitencourt et al. (2010) e Reboita et al. (2010) aplicaram a metodologia desenvolvida por Simmonds e Murray (1999) em dados da reanálise 1 do NCEP (National Center for Environmental Prediction) (KALNAY et al., 1996) para detectar ciclones extratropicais na costa centro-sul do país. O procedimento é eficiente para detectar baixas pressões fechadas relativas independente de sua intensidade (MURRAY; SIMMONDS, 1991 e BITENCOURT et al., 2010).

Uma análise interanual da frequência de ciclones extratropicais na costa sul do Brasil foi realizada em Mello et al. (2012). Porém, o objetivo do presente trabalho é investigar a ocorrência e o tempo de vida da variação sazonal e interdecadal dos ciclones extratropicais na costa sul do Brasil.

\section{Material e Método}

O presente trabalho utilizou a metodologia aplicada em Bitencourt et al. (2010) para obtenção de dados de ocorrência de ciclones entre os anos de 1957 a 2010. A área de seleção dos ciclones foi $15^{\circ} \mathrm{S}$ e $45^{\circ} \mathrm{S}$ e $20^{\circ} \mathrm{W}$ e $60^{\circ} \mathrm{W}$ (Fig. 1).

Foram considerados apenas os sistemas de baixas pressões fechadas à superfície, ou seja, com centros definidos de acordo com Simmonds e Murray (1999). Além disso, neste trabalho não se considera a diferença de intensidade dos ciclones. Foram selecionados os ciclones com tempo de vida igual ou superior a 24 horas. Desta forma, não foram quantificadas outras baixas transitórias de curta duração. Assim, determinou-se de forma objetiva o ciclo de vida dos ciclones, bem como o intervalo de ocorrência entre dois ciclones.

Apresenta-se, também, uma análise sazonal à ocorrência de ciclones pelas estações do ano (verão, outono, inverno, primavera).

O estudo sobre a variabilidade interdecadal da ocorrência de ciclones extratropicais foi realizado para três períodos referentes aos anos de 1957 a 2010: 1ํo período de 1957 a 1975 (maior ocorrência de La Niña); $2^{\circ}$ período de 1976 a 1999 (maior ocorrência de El Niño); e, 3º período de 2000 a 2010 (período mais recente).

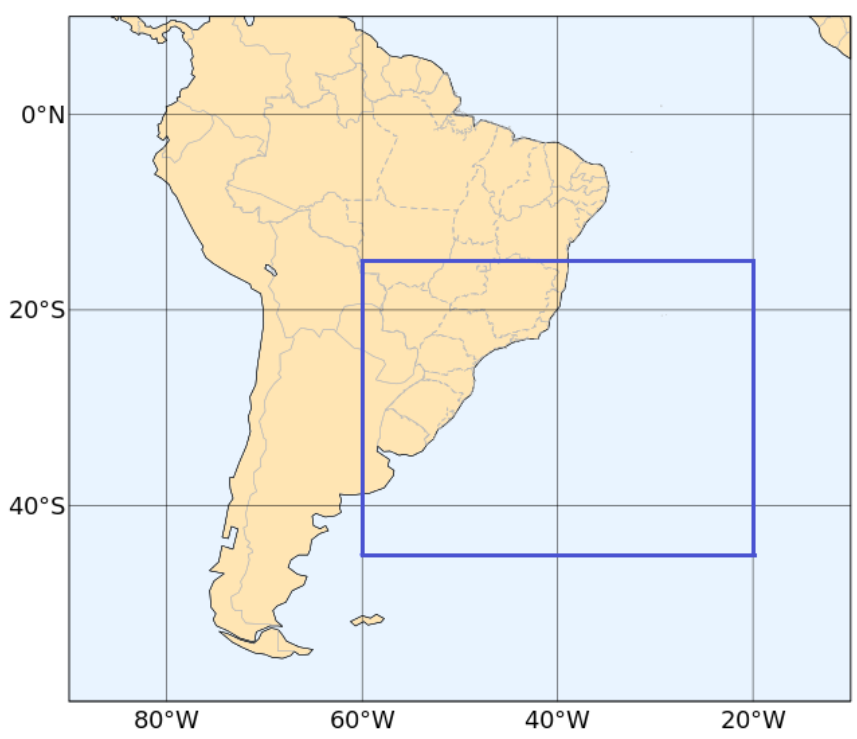

Figura 1 - Os ciclones extratropicais foram identificados dentro da uma área correspondente a latitudes de $15^{\circ} \mathrm{S}$ e $45^{\circ} \mathrm{S}$ e longitudes de $20^{\circ} \mathrm{W}$ e $60^{\circ} \mathrm{W}$, delimitada na figura

\section{Resultados e discussão}

A distribuição espacial dos ciclones extratropicais baseado nos três períodos interdecadais estabelecidos mostra a sua localização inicial, apresentando áreas preferenciais de ciclogênese. Na distribuição espacial, apresentada pela Fig. 2, pode-se observar que uma quantidade representativa de ciclones extratropicais ocorreu à leste do continente sul-americano, costa sul-brasileira, leste da Argentina e Uruguai, sendo esta uma região de forte atividade ciclogenética (GAN e RAO, 1994; REBOITA et al., 2012).

A quantidade de ciclones no período de 1957-1975 foi de 1090 ciclones (média de 57,37 por ano), no período de 1976-1999 foi de 1370 (média de 57,08 por ano) e no período de 2000-2010 foi de 661 (média de 60,09 por ano). Interessante observar que as regiões favoráveis à formação de ciclones (costa sul e sudeste do Brasil) são mais evidentes principalmente para os períodos de 1957-1975 (Fig. 2a) e 1976-1999 (Fig. 2b).

Quanto ao posicionamento médio dos ciclones extratropicais da pesquisa, o valor médio da longitude e da latitude é de aproximadamente $46^{\circ} \mathrm{W}$ e $30^{\circ} \mathrm{S}$, respectivamente. Murray e Simmonds (1991) apresentaram valor semelhante de latitude, entre $20^{\circ} \mathrm{S}$ e $30^{\circ} \mathrm{S}$.

a) 


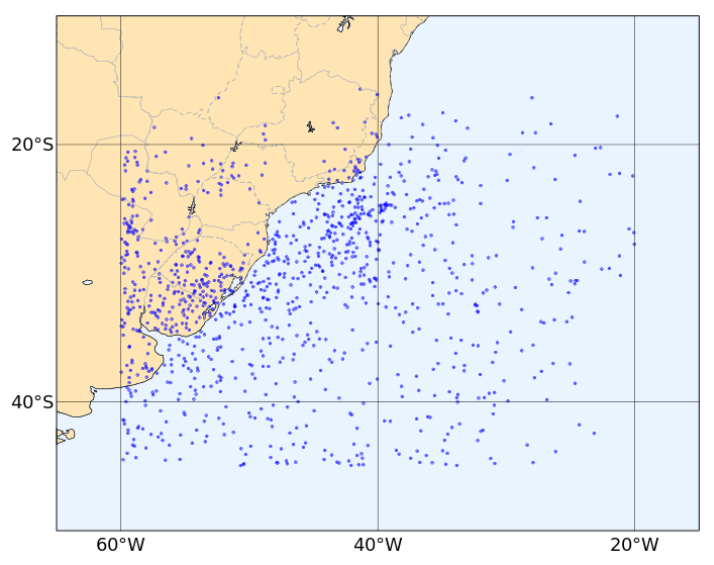

b)

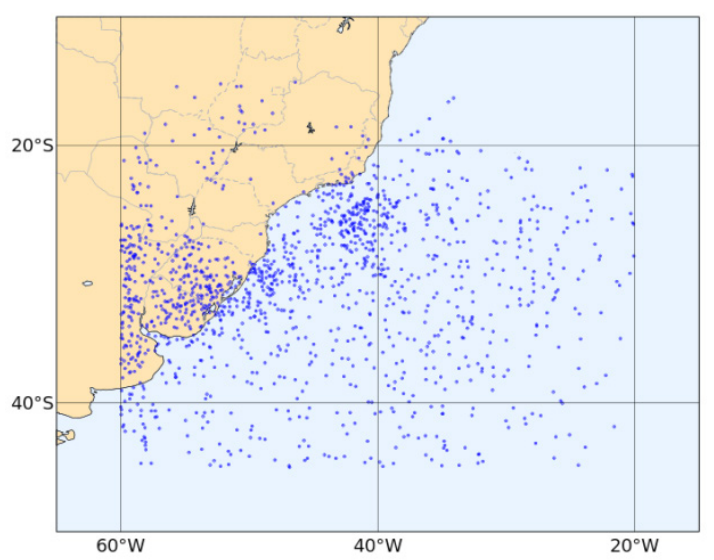

c)

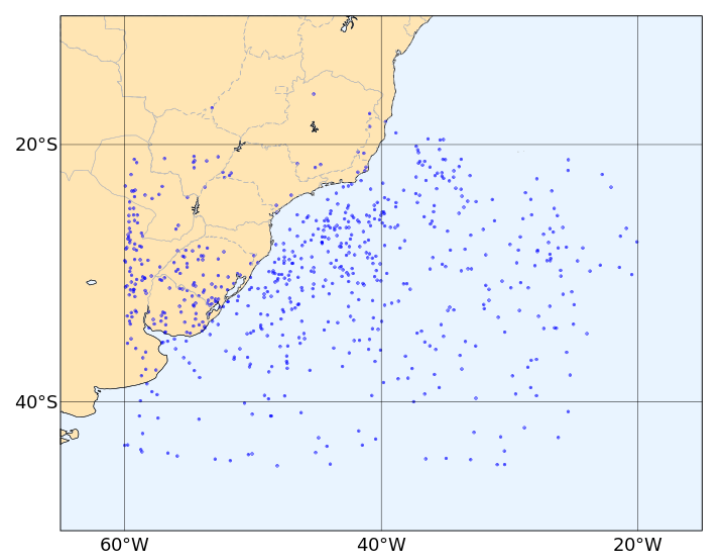

Figura 2 - Distribuição dos ciclones extratropicais de acordo com a sua localização inicial, em pontos, para os períodos de 1957-1975 (a), de 1976-1999 (b) e de 20002010 (c), respectivamente

A seleção da ocorrência de ciclones teve como objetivo estabelecer os principais casos, observando o tempo de vida de cada caso. Na Figura 3 é mostrada a frequência anual de ciclones no período em estudo. Os resultados mostraram um total de 3121 ciclones ao longo dos 54 anos analisados. $\mathrm{O}$ ano com maior ocorrência de ciclones extratropicais foi 2007 (Fig. 3c), com 74 ciclones; o ano com menor quantidade foi 1969 (Fig. 3b), com 47.

a)

1957-1975

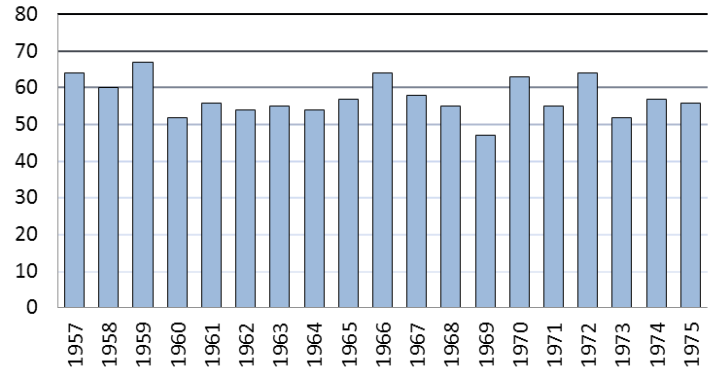

b)
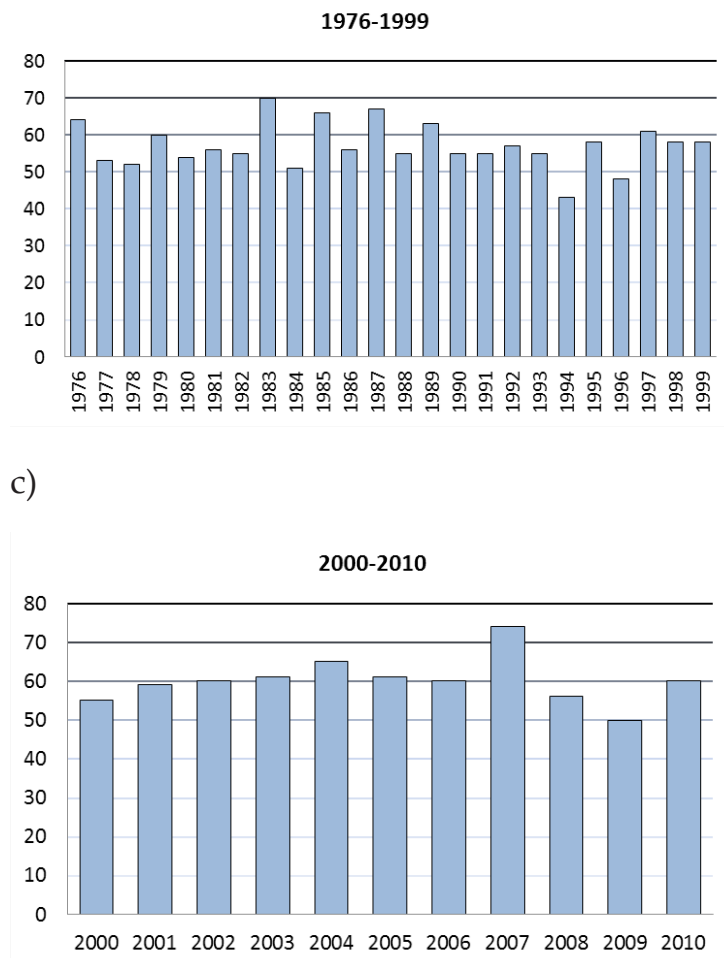

Figura 3 - Distribuição de frequência de ciclones extratropicais para os três períodos interdecadais da pesquisa 1957-1975 (a), 1976-1999 (b), 2000-2010 (c), respectivamente

A correlação entre a ocorrência de ciclones e os eventos do El Niño Oscilação Sul (ENOS) para todo o período analisado foi 0,134. A interação entre a ocorrência anual de ciclones extratropicais (eventos de escala sinótica) com o El Niño Oscilação Sul (ENOS) foi investigada com o coeficiente de correlação linear entre a anomalia da mediana da quantidade de ciclones a cada ano e a mediana do índice do ENOS (CPC-NCEP, 2013) de cada ano, resultando em um coeficiente positivo e mostrando uma fraca relação. Entretanto, Pezza e Ambrizzi (2003) 
mostraram que em anos de La Niña o Atlântico Sudoeste apresenta os ciclones mais intensos.

A seleção de ciclones possibilitou um cálculo do tempo de vida de cada evento. Observou-se que em 35,23\% dos casos, o tempo de vida foi de 2 dias. Porém há um caso que se destaca, com 10 dias de duração, iniciado em 8 de agosto 2003. A Figura 4 mostra o percentual da persistência de ciclones extratropicais por dia de duração. Nota-se que em $86,87 \%$ dos casos os ciclones tiveram um tempo de vida de até 3 dias. Assim, 13,13\% ultrapassaram os 3 dias de duração. Esta seleção de ciclones extratropicais de acordo com seu ciclo de vida permitiu apontar o tempo de vida superior a média apontada por Murray e Simmonds (1991).

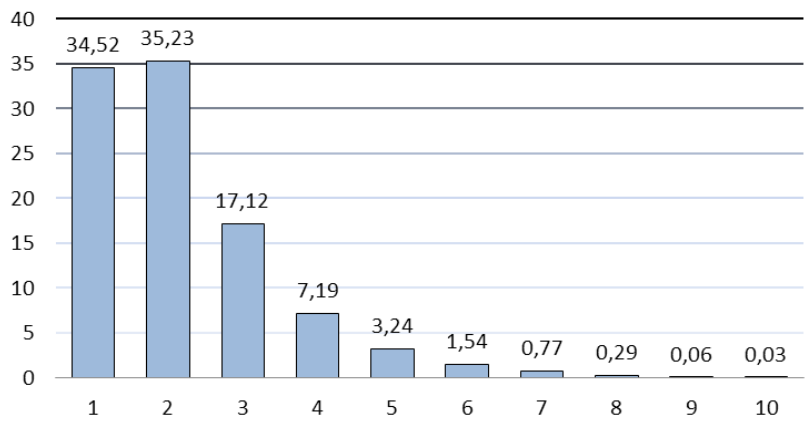

Figura 4 - Tempo de vida de ciclones em percentual com relação ao tempo de permanência de cada evento, de 1 a 10 dias

Com isso, esta pesquisa apresenta também a duração dos intervalos entre os ciclones selecionados, calculandose a diferença temporal entre um ciclone extratropical e o posterior. Isto é, foi possível analisar a duração do intervalo entre dois ciclones.

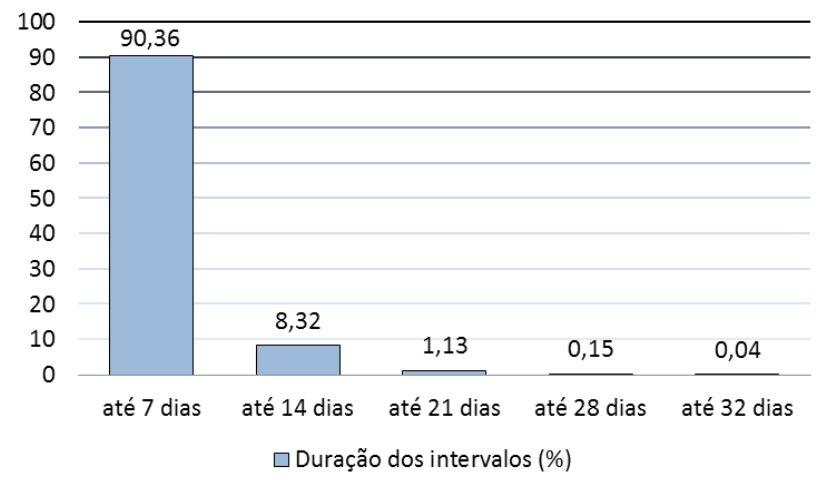

Figura 5 - Persistência dos intervalos em percentual (\%). Percentuais acumulados de 1 a 7 dias, de 1 a 14, de 1 a 21 dias e assim por diante

Dos ciclones selecionados na área de $15^{\circ} \mathrm{S}$ e $45^{\circ} \mathrm{S}$ e $20^{\circ} \mathrm{W}$ e $60^{\circ} \mathrm{W}, 50 \%$ deles ocorreram em intervalos de 1 dia. A Figura 5 apresenta o histograma da duração dos intervalos para todo o período analisado. Nota-se que mais de $90,36 \%$ dos ciclones atingem o Sul do Brasil em intervalo de até 7 dias. Períodos longos, sem ocorrência do sistema na região, são menos frequentes e intervalos superiores a 10 dias envolvem menos de 5\% dos casos analisados. O intervalo máximo encontrado foi de 32 dias entre 24 de fevereiro e 28 de março de 1964. Além disso, para intervalos acima de 14 dias encontrou-se 70 casos em 54 anos de dados.

Com relação à sazonalidade - em concordância com Gan e Rao (1994) - a estação com maior ocorrência de ciclones é o inverno, com 822 ciclones, entre os anos de 1957 a 2010. A média de ciclones extratropicais para este período no inverno é de 15,22 . O outono foi a estação com menor incidência de ciclones para o período, 759 ciclones (média de 14,06).

A Figura 6 apresenta a média de ciclones extratropicais para os períodos interdecadais escolhidos (19571975; 1976-1999; 2000-2010), nas quatro estações do ano. Observa-se a variabilidade interdacadal que, entre 1957-2010 o inverno foi a estação com maior incidência de ciclones, já entre 2000-2010 o outono foi a estação com maior incidência.

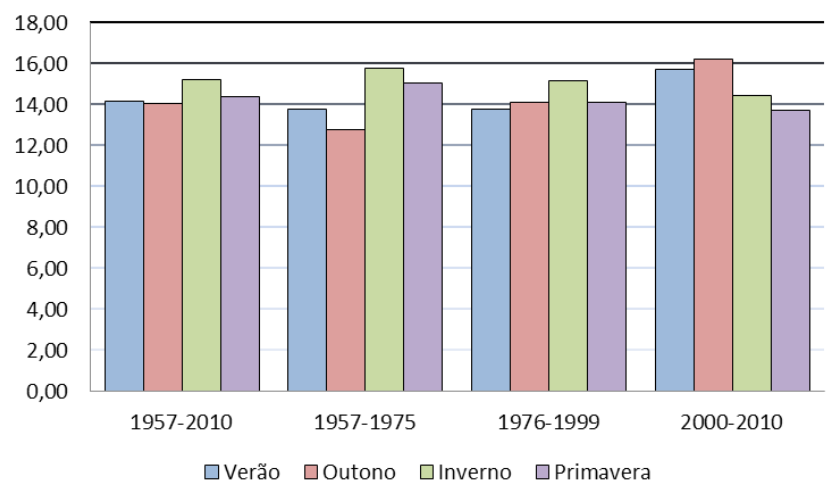

Figura 6 - Médias das quantidades de ciclones para cada um dos períodos analisados, em cada estação do ano (verão, outono, inverno e primavera)

A média de ciclones é semelhante em todas as categorias (Fig. 6). Está é a hipótese nula na análise de significância estatística. Para inferir se esses resultados são estatisticamente consistentes foi usado o teste de diferença de duas proporções (WILKS, 2007). A hipótese de que esta diferença é significativa foi verificada usando teste $t$ de Student. Por exemplo, verificou-se a diferença entre a maior ocorrência de ciclones no inverno na categoria do período de 1957-2010. A hipótese que a maior ocorrência é significativa estatisticamente pode ser aceita ao nível de $90 \%$ do referido teste, mas não para 95\%. Nota-se também um aumento da ocorrência de ciclones no verão e outono em comparação ao inverno e à primavera no período de 2000-2010, que é significativa ao nível de $95 \%$ de confiança.

Além disso, pode-se observar que entre 1957 a 1975 (Fig. 6) havia maior ocorrência de ciclones no inverno 
e primavera comparativamente ao verão e outono com significância estatística ao nível de 5\%. A hipótese de tendência de aumento de ocorrência de ciclones no outono e uma diminuição no inverno e primavera dos períodos interdecadais estudados pode ser aceita com significância de $90 \%$, mas não para $95 \%$.

A elaboração desta análise, sazonal e interdecadal de ciclones, se faz relevante devido à importância destes sistemas para o tempo e clima da região subtropical do país. Cardoso et al. (2012) mostram que a estes sistemas meteorológicos estão ligados à ocorrência de precipitação, ventos fortes e agitação marítima. Portanto, este trabalho corrobora com o conhecimento da variabilidade de atuação dos ciclones extratropicais no Atlântico Sudoeste.

\section{Conclusão}

As baixas pressões relativas a dados da reanálise 1 do NCEP estudadas nesta pesquisa possibilitaram uma análise do ciclo de vida dos ciclones extratropicais e mostraram que em $86,87 \%$ dos casos os ciclones tiveram um ciclo de vida de 3 dias. Entretanto, 90,36\% dos ciclones atingem a região Sul do Brasil em intervalos de 7 dias.

A distribuição espacial mostrou que grande parte dos ciclones extratropicais neste período ocorreu ao longo da costa brasileira. A porção continental com ocorrência representativa de ciclones é o estado do Rio Grande do Sul, Uruguay e leste da Argentina - local de grande atividade ciclogenética.

Este estudo mostra a ocorrência dos ciclones associados a variabilidade sazonal a interdecadal dos ciclones nesta região. A média dos ciclones extratropicais nos diferentes períodos sazonais do ano mostra que de 1957 a 2010, o inverno foi o período de maior ocorrência, com $26,34 \%$ do total, na qual há média de 15,22 ciclones por inverno. Porém, há uma tendência significativa de aumento da média dos ciclones extratropicais no verão -outono quanto comparados os períodos de 1957-1975 para 2000-2010.

\section{Agradecimentos}

Os autores agradecem o Dr. Daniel Bitencourt por ter cedido os dados a esta pesquisa, tal como ao IFSC por ter possibilitado a realização desta pesquisa. $\mathrm{O}$ autor agradece, também, ao Grupo de Desenvolvimento em Assimilação de Dados, da Divisão de Modelagem e Desenvolvimento do CPTEC/INPE, por possibilitar a apresentação do mesmo no V Simpósio Internacional de Climatologia, da Sociedade Brasileira de Meteorologia, em Florianópolis/SC.
BITENCOURT, D. P. et al. Relating winds along the Southern Brazilian coast to extratropical cyclones. Meteorological Applications, Londres, v. 18, n. 2, p.223229, 17 set. 2010.

CARDOSO, C.S.; BITENCOURT, D.P.; MENDONÇA, M. Comportamento do vento no setor leste de Santa Catarina sob influência de ciclones extratropicais. Revista Brasileira de Meteorologia, São Paulo, v. 27, n. 1, p.3948, mar. 2012. Trimestral.

Climate Prediction Center - National Centers for Environment Prediction (CPC-NCEP). Cold and Warm Episodes by Season. Oceanic Niño Index (ONI). Disponível em:

<http://www.cpc.ncep.noaa.gov/products/analysis monitoring/ensostuff/ensoyears.shtml>. Acesso em: 20 ago. 2013.

GAN, M. A.; RAO, Vadlamudi Brahmananda. The influence of the Andes Cordillera on Transient Disturbances. Monthly Weather Review, Washington, v. 122, n. 6, p.1141-1157, jun. 1994. Mensal.

KALNAY, E. et al. The NCEP/NCAR 40-Year Reanalysis Project. Bulletin Of The American Meteorological Society, Washington, v. 77, n. 3, p.437-471, mar. 1996.

MELLO, M. G.; SILVA, M. S.; MUZA, M.N. Análise interanual de ciclones no litoral brasileiro, de 1957 a 2010. In: XVII Congresso Brasileiro de Meteorologia, 2012, Gramado/RS. Anais XVII Congresso Brasileiro de Meteorologia. Disponível em:

<http://www.cbmet2012.com/anais/pdfs/4XKK.pdf $>$. Acesso em: 05 jan. 2013.

MURRAY, R. J.; SIMMONDS, I. A numerical scheme for tracking cyclone centers from digital data. Part I: development and operation of the scheme. Australian Meteorological Magazine, Melbourne, v. 39, n. 3, p.155166, jul. 1991.

PEZZA, A. B.; AMBRIZZI, T. Variability of Southern Hemisphere Cyclone and Anticyclone Behavior: Further Analysis. Journal Of Climate, Washington, v. 16, n. 7, p.1075-1083, abr. 2003.

REBOITA, M. S.; ROCHA, R. P.; AMBRIZZI, T. Climatologia de Ciclones sobre o Atlântico Sul Utilizando Métodos Objetivos na Detecção destes Sistemas. In: IX CONGREMET, Congresso Argentino de Meteorologia, Buenos Aires, AR, Outubro 3-7, 2005.

REBOITA, M. S. et al . Regimes de precipitação na América do Sul: uma revisão bibliográfica. Revista Brasileira de

\section{Referências}


Meteorologia, São Paulo, v. 25, n. 2, jun. 2010. Trimestral.

REBOITA, M. S.; KRUSCHE, N.; AMBRIZZI, T. e ROCHA, R. P. Entendendo o Tempo e o Clima na América do Sul. Terrae Didatica, Campinas, vol.8, n.1, pp. 34-50. 2012. Semestral.

SELUCHI, M. E. Diagnóstico y prognóstico de situaciones sinópticas conducentes a ciclogénesis sobre el este de Sudamérica. Geofísica Internacional, México, v. 34, n. 2, p.171-186, 1995. Trimestral.

SIMMONDS, I.; MURRAY, R. J.. Southern Extratropical Cyclone Behavior in ECMWF Analyses during the FROST Special Observing Periods. Weather And Forecasting, Washington, v. 14, n. 6, p.878-891, 22 mar. 1999. Bimestral.

WILKS, D. S.. Statistical methods in the atmospheric science. 2. ed. San Diego: American Press, 2006. ISBN 978-0-12-751966-1. 